\title{
Multi-scale and multimodal $x$-ray microscopy and applications
}

\author{
Xianghui Xiao
}

\section{Brookhaven National Laboratory, Upton, New York, United States}

X-ray microscopy and imaging have been utilized to study the heterogeneity in complex systems, e.g., batteries. The microscopic structural information reveals the variant local structure conformation and properties so complements the average information at the macroscopic level obtained with other techniques.

While X-ray microscopy and imaging applications are growing rapidly in the recent years, there are general challenges associated to the nature of these high-resolution techniques. Data statistics and sample radiation damage are two outstanding issues. Multimodal techniques at multiscales provide options to mitigate these problems.

Transmission X-ray Microscopes (TXM) is a full-field X-ray nano-imaging technique. Comparing to the nanobeam scanning probe techniques, it has advantages of high throughput. TXM can acquire billions of X-ray absorption spectra of a sample in short time. Therefore, the good data statistics help to reduce the bias due to limited number of measurements. This capability makes it a powerful tool in studying energy storage materials. The position sensitive structural information reveals the good and the bad working modes in the heterogeneous system and is helpful to improve the system engineering designs to prompt the good modes and suppress the bad modes.

Although TXM is capable to provide correlative spectro-morphological imaging capabilities, other scanning nano-probe techniques are needed to pursuit even more modalities at higher sensitivity. At a state-of-art nanoprobe beamline, nano-diffraction, nano-fluorescence, and scanning imaging techniques can be integrated onto a same platform. Multimodal measurements with these techniques provide correlative structural information of the samples from different aspects. However, such measurements are slow, so it is not suitable for in situ and high throughput experiments. Combining TXM and nano-probe techniques overcomes each technique's limitations and provides deep insights of the measured system. Few examples of this approach will be discussed in the presentation.

\section{References}

1. "Transmission X-ray Microscopy and Its Applications in Battery Material Research - A Short Review," S. Spence, W.K. Lee, F.Lin , X. Xiao, submitted

2. "Charge distribution guided by grain crystallographic orientations in polycrystalline battery materials," Z. Xu, Z. Jiang, C. Kuai, R. Xu, C. Qin, Y. Zhang, M. M. Rahman, C. Wei, D. Nordlund, C. J. Sun, X. Xiao, X. W. Du, K. Zhao, P. Yan, Y. Liu, F. Lin, Nat. Commun. 11 83, 2020

3. "Charging Reactions Promoted by Geometrically Necessary Dislocations in Battery Materials Revealed by In Situ Single-Particle Synchrotron Measurements," Z. Xu, D. Hou, D. J. Kautz, W. Liu, R. Xu, X. Xiao, F. Lin, Adv Mater 32, 2003417, 2020 